\title{
Effects of Deep Cervical Flexor Exercise with Visual Guide on Muscle Activity and Craniovertebral Angle in Subjects with Forward Head Posture
}

\author{
Kuk-kyung Son, PT. • Heon-Seock Cynn, $\mathrm{PT}, \mathrm{PhD}^{\dagger} \cdot$ Ji-Hyun Lee, PT, PhD ${ }^{1}$ \\ Dong-Hwan Park, PT, MS • Bo-Been Kim, PT, MS \\ Department of Physical Therapy, Graduate School, Yonsei University \\ ${ }^{1}$ Department of Physical Therapy, Baekseok University
}

Received: March 4, 2019 / Revised: March 4, 2019 / Accepted: March 13, 2019

(c) 2019 J Korean Soc Phys Med

\section{| Abstract |}

PURPOSE: Forward head posture (FHP) is a head-on-trunk malalignment that results in musculoskeletal dysfunction and neck pain. To improve forward head posture, both the craniocervical flexion exercise (CCFE) and the visual guide (VG) technique have been used. This study compared the immediate effects of CCFE and VG combined with CCFE on craniovertebral angle (CVA), as well as on the activity of the sternocleidomastoid (SCM) and anterior scalene (AS) muscles during CCFE in subjects with FHP.

METHODS: In total, 16 subjects (nine males, seven females) with FHP were recruited using the G-power software. Each subject conducted CCFE and CCFE combined with VG in random order. The CVA was recorded using a digital camera and the ImageJ image analysis software. The EMG data of SCM and AS were measured by surface electromyography. A paired T-test was used to assess

†Corresponding Author : Heon-Seock Cynn cynn@yonsei.ac.kr, http://orcid.org/0000-0002-5810-2371

This is an Open Access article distributed under the terms of the Creative Commons Attribution Non-Commercial License (http://creativecommons.org/licenses/by-nc/3.0) which permits unrestricted non-commercial use, distribution, and reproduction in any medium, provided the original work is properly cited. differences between the effects of the CCFE and VG combined with CCFE interventions in the same group.

RESULTS: The CVA was significantly greater for CCFE combined with the VG than for CCFE alone $(p<.05)$. The activity of the SCM and AS muscles was also significantly greater when the VG was combined with CCFE than during CCFE alone across all craniocervical flexion exercise phases $(\mathrm{p}<.05)$.

CONCLUSION: Use of the VG technique combined with CCFE improved FHP in subjects with FHP compared to CCFE alone.

Key Words: Forward head posture, Craniocervical flexion exercise, Visual guide

\section{Introduction}

Forward head posture (FHP) is a postural head on trunk misalignment associated with pain in the neck and shoulder region [1]. FHP is often explained as excessive forward positioning of the head relative to a vertical reference line that involves increased cervical spine lordosis and rounded shoulders with thoracic kyphosis [2]. Individuals with FHP are generally associated with weakness of the deep cervical flexors (DCFs) (i.e., longus colli and capitis) and mid- 
thoracic scapular retractors (i.e., middle and lower trapezius, rhomboids and serratus anterior), as well as shortening of the opposing cervical extensors and pectoralis muscles [2,3]. Prolonged FHP posture can cause decreased craniocervical flexion range of motion (ROM) and activation of the deep neck flexors and is associated with increased activation of the superficial muscles, such as anterior scalene (AS) and sternocleidomastoid muscle (SCM) [4]. In addition, FHP has been associated with cervical pain, fatigue, and limited range of motion in the neck and shoulder, as well as discomfort symptoms caused by excessive loading of the cervical spine [5].

The general consensus of the cause of FHP is that it is the result of habitual posture patterns during working. Thus, correction exercises are prescribed to improve head on trunk misalignment in FHP [1,6], particularly craniocervical flexion exercise (CCFE). FHP exercise programs include strengthening of DCFs and the shoulder retractor and stretching of pectoral muscles and the cervical extensor. The activation of DCFs is increased and the cervical extensor is stretched during the CCFE; therefore, it improves the ability to hold an upright cervical spine posture [7]. In a previous study, CCFE was found to effectively increase the activation of DCFs and decrease the activation of SCM and AS in people with chronic neck pain [4].

Control of the cervical muscles is complicated because it requires to the integrated movement and stability of the major sensory organs of vision, hearing and balance with movement and stability of the head and neck. Using a visual guide (VG), the activity of the global and local neck muscles were shown to be affected by movements of the eyeball [8]. In neck rotation with eyeball movement, activity of the SCM, right splenius capitis (SC), and multifides (MF) changed. Additionally, when subjects gazed $45^{\circ}$ towards the right $\mathrm{SC}$ with neck rotation, left SCM and right splenius capitis (SC) EMG activity increased [8]. This is thought to have occurred because of the interaction between the rotators of the neck and the extra-ocular eye muscles for the eye and head coordinated movement [9].

Various VG will affect activation of neck muscles including the $\mathrm{SCM}, \mathrm{SC}, \mathrm{MF}$ and $\mathrm{AS}$ muscles during neck movement. A smaller craniovertebral angle (CVA) is associated with a greater FHP. Although high reliability of this procedure $(\mathrm{ICC}=.880)$ has previously been reported [10], no studies have investigated the effects of VG on CVA and the amplitude of muscle activity of SCM and AS during CCFE in subjects with FHP. Therefore, the present study investigated the immediate effects of VG on the CVA and the muscle activity of SCM and AS during CCFE in subjects with FHP. We hypothesized that the CVA would increase as would the amplitude of muscle activity of SCM and AS when VG was provided.

\section{Methods}

\section{Subjects}

To conduct power analysis, a pilot study with five subjects was performed. A total sample size of 16 subjects was required to satisfy a level of .05 , power of .80 , and effect size of .655 (G-power software 3.1.2; Franz Faul, University of Kiel, Kiel, Germany). The subjects (12 males, 7 females) were 19 young adults with FHP who volunteered to participate in this study (age $=21.789 \pm 1.228$ years, height $=170.789 \pm 8.541 \mathrm{~cm}$, weight $=66.036 \pm 10.348 \mathrm{~kg}$, body mass index $=22.546 \pm 2.136$, CVA before interventions $=$ $45.680 \pm 5.987^{\circ}, 17$ right and 2 left side). The only specific inclusion criterion for participation was that the CVA be $<53^{\circ}[11]$

The exclusion criteria were: (1) medical/health care for neck, shoulder, or lower back pain over the past year [1], (2) musculoskeletal pain, dysfunction of the spine, and fractures and abnormalities in cervical, thoracic or shoulder girdle [12], (3) fat determined by body mass index $>30$ $\mathrm{kg} / \mathrm{m}^{2}$ [13] and (4) vestibular disorder, asthma, previous 
severe trauma to the head or neck, or disorders of the eye [8]. Prior to starting data collection, all subjects were made aware of the experimental protocol from the principal investigator (PI). The Yonsei University Wonju Institutional Review Board approved the study and all subjects signed an informed consent form.

\section{Measurement}

\section{1) CVA}

The CVA was recorded using a digital camera (Samsung, Seoul, Korea) and the ImageJ image analysis software (National Institutes of Health, USA) was used to assess the kinematics data. The digital camera was placed perpendicular to the ground with its lens $80 \mathrm{~cm}$ from the lateral aspect of the subject and pointing directly at the subject's shoulder to minimize parallax error [14]. The subject sat on a stool that was placed in the reference area in a natural and relaxed pose. The subject was asked to put both feet on the ground and place their hands on their thighs with a relaxed back. Next, the PI instructed the subject to fix their gaze on a marked point directly on the wall. The PI then attached a body marker to the external auditory meatus of the ear and another to the spinous process of the $\mathrm{C} 7$ [15]. Next, two photographs were taken with the subject's upper body in the lateral aspect of the dominant side with a flash. After the first photograph was taken, the subject was asked to stand and then sit again. The CVA was determined between the line from the external auditory meatus to the $\mathrm{C} 7$ line and a horizontal line through the $\mathrm{C} 7[14,15]$ (Fig. 1).

\section{2) Surface electromyography recording and data processing}

Surface electromyography (EMG) data were collected using a Tele-Myo DTS EMG instrument with a wireless telemetry system (Noraxon, Inc., Scottsdale, AZ, USA) and analyzed by the Noraxon MyoResearch 1.06 software. Data

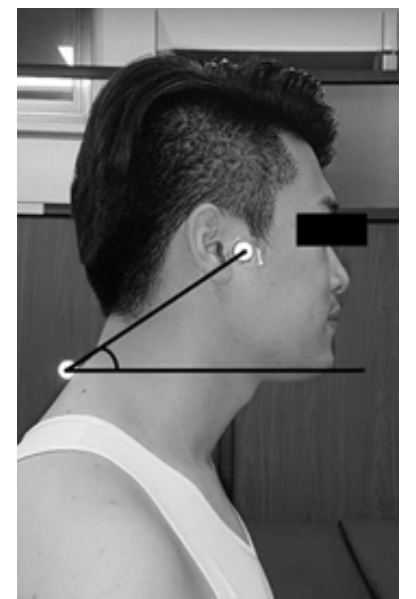

Fig. 1. Measurement of the craniovertebral angle (CVA)

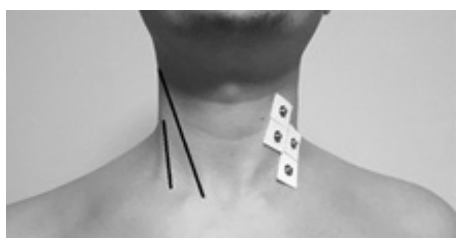

Fig. 2. Electrodes of surface electromyography (EMG) in the sternocleidomastoid (SCM) and anterior scalene (AS)

were recorded from the SCM and AS on the subjects' dominant side. The EMG signals were amplified, band-pass filtered $(20-450 \mathrm{~Hz})$, and notch filtered $(60 \mathrm{~Hz})$. The data were then recorded using a $1000 \mathrm{~Hz}$ sampling rate and processed into root-mean-square values with a window of $50 \mathrm{~ms}$. To reduce impedance to the EMG signal, the electrode sites were shaved, after which the skin was cleaned and rubbed with alcohol using a sterile gauze pad. Bipolar electrodes $(\mathrm{Ag} / \mathrm{AgCl})$ were then adhered to the skin with a $2 \mathrm{~cm}$ inter-electrode distance in the direction of the muscle. The electrodes in SCM and AS were placed as follows: SCM, slightly posterior from the middle of the distance between the mastoid process and the sternal notch; AS, slightly oblique angle just posterior from the SCM, immediately above the clavicle and anterior to the upper trapezius in the hollow triangle [16] (Fig. 2). The 
EMG data for the SCM and AS were normalized using the maximum voluntary isometric contraction (MVIC). To collect the MVIC data, standard manual muscle-test positions were used as follows: SCM and AS, supine, flexion of the elbow and hands beside the head, anterolateral flexion of the neck with the face turned toward the non-tested side [17]. The mean value of the two trials was taken for data analysis and the mid three seconds of each trial were used. Subjects performed and held the position for five seconds with a three minute rest between muscle contractions. The intra-class correlation coefficients (ICC) for the MVICs of SCM and AS were 989 (95\% Confidence Interval [CI]: .944-.992) and .965 (95\% CI: .834-.973), respectively. The EMG activity for the SCM and AS during the CCFE was expressed as a percentage of the mean MVIC (\%MVIC)

\section{Procedure}

CCFE and CCFE-VG were used for the intervention. To prevent carryover effects, randomization was conducted using a website (http://www.randomization.com) to determine the sequence of intervention. All intervention procedures were performed by the second investigator. After one intervention was performed, the subject took a 20 min rest to minimize any carry-over effect between interventions. All measurements were made twice by the principal investigator immediately after each intervention, and the mean of the two measurements was used for statistical analysis. The principal investigator took the measurement in a separate space to ensure that neither the investigators nor the subject were biased from the results. The other investigator who analyzed the measurement data was blinded to the experimental conditions.

\section{1) $\mathrm{CCFE}$}

CCFE is a low load exercise of the cranio-cervical flexors in which the DCFs of the upper cervical part (longus capitis and longus colli) are contracted without recruitment of the superficial flexor muscles (sternocleidomastoid and anterior scalene). The protocol for CCFE was established from previous studies $[4,18]$. In the first phase of the exercise, the PI taught the subject to perform controlled CCFE slowly in the supine position. The subject focused on sagittal rotation movement of the head slide in caudad and cephalad directions on the bed rather than a retraction movement. Once the first CCFE was achieved correctly, the subject performed the second phase of the exercise using a pressure biofeedback unit (Stabilizer ${ }^{\mathrm{TM}}$, Chattanooga Group Inc. USA) between the back of the head and bed. In the third phase, the subjects were asked to perform progressing ranges of CCFE by increasing the amount of pressure on the feedback dial, flattening the cervical lordosis. In the fourth phase, the subject performed CCFE gradationally to reach the fifth phase of pressure target levels from $20 \mathrm{mmHg}$ to $30 \mathrm{mmHg}$ in $2 \mathrm{mmHg}$ increments. The PI confirmed that the subject could hold the target level consistently for 10 seconds without depending on retraction, dominant contraction of the superficial neck flexor muscles, or a jerk and quick CCFE movement. Recruitment of the superficial muscles was monitored by the PI using palpation. The contraction time was 10 seconds with 10 repetitions and the subject had 3-5 seconds of rest periods between each contraction at each target level [4] (Fig. 3).

\section{2) CCFE-VG}

The procedure of CCFE-VG is the same as that for

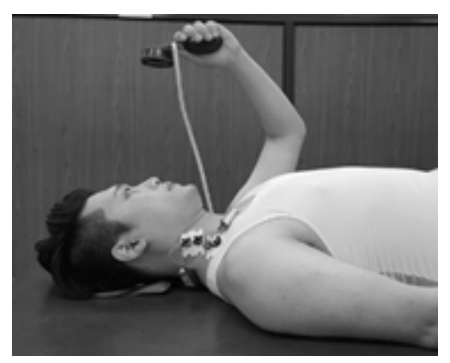

Fig. 3. Cranio-cervical flexion exercise (CCFE) 


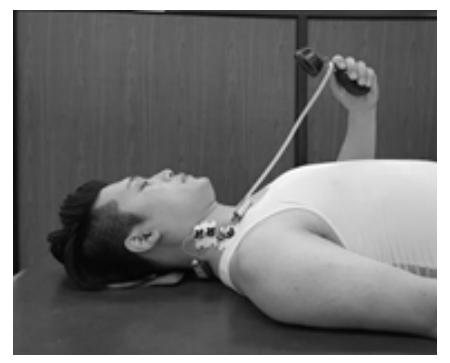

Fig. 4. Cranio-cervical flexion exercise with visual guide (CCFE-VG)

CCFE, except for the addition of VG. In the first phase, the PI explained the CCFE-VG protocol in detail and taught the subject to perform controlled downward eyeball movement (CDEM) slowly in the supine position without palpable contraction of the SCM and AS or movement of the cervical flexion. In the second phase of CCFE-VG, the PI stood by the subjects, touched their SCM and AS in the supine position and asked them to look straight ahead and then lower their eyeball as slowly as possible. Once the CDEM without cervical flexion was achieved correctly, the PI taught the subjects to perform controlled CCFE slowly in the third phase. In the fourth phase, the PI asked the subjects to perform CCFE and joined CDEM in each target pressure level slowly and precisely. When these protocols proceeded successfully, the fifth phase began, in which the subjects performed CCFE using a pressure biofeedback unit (Stabilizer ${ }^{\mathrm{TM}}$, Chattanooga Group Inc. USA) between the back of the head and the bed. The subjects were asked to look forward and to hold and watch the feedback dial to identify the pressure level. In the sixth phase, the subjects performed CDEM during the CCFE slowly so that it could coincide with CCFE in a gradational process to reach the fifth phase of target pressure levels from a baseline of $20 \mathrm{mmHg}$ to the final level of $30 \mathrm{mmHg}$ in $2 \mathrm{mmHg}$ increments. The PI stood by the subjects and supervised them while they held the target pressure level steadily for 10 seconds without retraction and dominant use of the cervical flexor muscles, then performed CDEM in order at the same time. The contraction time was 10 seconds with 10 repetitions. At each target level, the subjects had 3-5 seconds of rest time (Fig. 4).

\section{Statistical analysis}

A one-sample Kolmogorov-Smirnov test was used to confirm the assumption of normal distribution. A paired-t test was used to measure the significance of differences in the CVA following CCFE and CCFE-VG intervention and in the activity of the SCM and AS muscles during CCFE and CCFE-VG in the same group with FHP. The level of significance was set at .05 . The effect sizes were calculated using Cohen's $d$ to determine meaningful changes, which is defined as differences between the interventions and mean CCFE values divided by the CCFE $\mathrm{SD}$, where an effect size of $\leq .10$ indicates a very small change, .20 a small change, .50 a moderate change, .80 a large change, 1.20 a very large change, and 2.0 the largest change [19]. Therefore, treatment results showing a large effect size indicate more meaningful outcomes than those showing a small effect size. All statistical analyses were performed using the Statistical Package for Social Science 21 (SPSS, Inc., Chicago, IL, USA).

\section{Results}

\section{CVA}

The CVA was significantly greater after CCFE-VG $\left(53.380 \pm 5.084^{\circ}\right)$ than after CCFE $\left(50.613 \pm 4.950^{\circ}\right)(\mathrm{p}<.05$, $\mathrm{ES}=.551$; Table 1, Fig. 5).

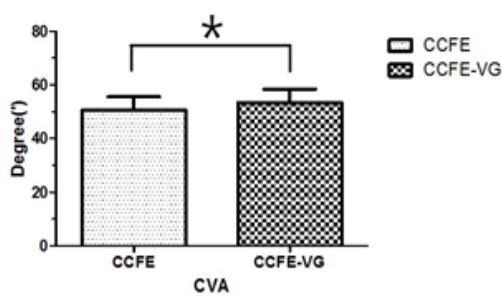

Fig. 5. Craniovertebral angle (CVA) after the craniocervical flexion exercise with visual guide (CCFE-VG) and the craniocervical flexion exercise (CCFE) 
Table 1. Comparison of CVA before the test and after CCFE and CCFE-VG

\begin{tabular}{cccc}
\hline & Pre-test & Post-test & P \\
\hline CCFE & $45.680 \pm 5.987^{\mathrm{a}}$ & $50.613 \pm 4.950$ & $.000^{*}$ \\
CCFE-VG & $45.680 \pm 5.987$ & $53.380 \pm 5.084$ & $.000^{*}$ \\
\hline
\end{tabular}

${ }^{a}$ Mean \pm standard deviation, ${ }^{*} \mathrm{p}<.05$

CCFE: craniocervical flexion exercise, CCFE-VG: the craniocervical flexion exercise with visual guide

$\mathrm{CVA}=$ Craniovertebral angle

Table 2. Comparison of SCM and AS during CCFE and CCFE-VG

(Unit: \%MVIC)

\begin{tabular}{ccccccc}
\hline & CCFE (SCM) & CCFE-VG (SCM) & P & CCFE (AS) & CCFE-VG (AS) & P \\
\hline Phase1 & $1.513 \pm 1.305^{\mathrm{a}}$ & $2.342 \pm 1.852$ & .032 & $3.850 \pm 2.512$ & $5.959 \pm 3.839$ & .008 \\
Phase2 & $2.026 \pm 1.880$ & $2.890 \pm 2.183$ & .001 & $4.719 \pm 2.944$ & $7.885 \pm 5.829$ & .007 \\
Phase3 & $2.537 \pm 2.557$ & $3.611 \pm 3.204$ & .009 & $6.918 \pm 6.059$ & $9.889 \pm 8.573$ & .004 \\
Phase4 & $3.615 \pm 4.877$ & $4.924 \pm 5.338$ & .006 & $8.983 \pm 8.907$ & $12.842 \pm 11.005$ & .000 \\
Phase5 & $4.336 \pm 6.196$ & $5.765 \pm 6.761$ & .010 & $11.264 \pm 12.676$ & $15.282 \pm 13.902$ & .000 \\
\hline
\end{tabular}

${ }^{a}$ Mean \pm standard deviation, ${ }^{*} \mathrm{p}<.05$

CCFE: craniocervical flexion exercise, CCFE-VG: the craniocervical flexion exercise with visual guide SCM: sternocleidomastoid, AS: anterior scalene
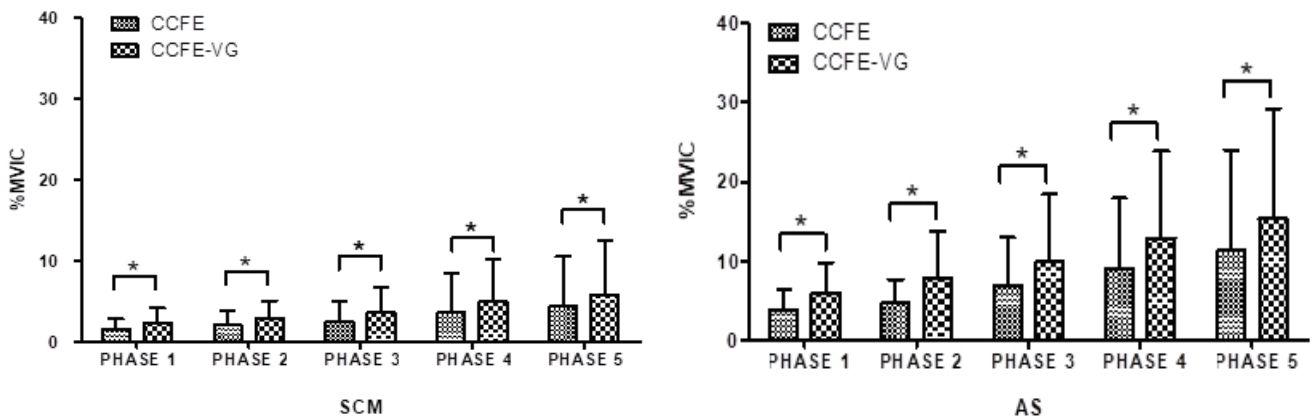

Fig. 6. Activity of the sternocleidomastoid (SCM) and anterior scalene (AS) muscles during the craniocervical flexion exercise (CCFE) and the craniocervical flexion exercise with visual guide (CCFE-VG)

\section{Muscle activity}

The activity of the SCM and AS muscles increased significantly with CCFE-VG compared to that with CCFE across all CCFE phases (all p<.050, ES: SCM Phase 1 $=.543$, Phase $2=.477$, Phase $3=.459$, Phase $4=.368$, Phase $5=.249$, AS Phase $1=.762$, Phase $2=.760$, Phase $3=.528$, Phase 4=.493, Phase 5=.344; Table 2, Fig. 6).

\section{Discussion}

The primary goal of this study was to compare the immediate effects of CCFE and CCFE-VG on (1) CVA and (2) the activity of the SCM and AS muscles in subjects with FHP. Comparison of CCFE and CCFE-VG measures showed that the CVA increased significantly in CCFE-VG compared to CCFE alone. In addition, the activity of the 
SCM and AS muscle increased significantly with CCFE-VG compared to CCFE across all CCFE phases. Our research hypothesis was supported by the results of this study.

CVA increased significantly in CCFE-VG compared to CCFE in this study. The CVA indicates the degree of FHP with an increased CVA reflecting an improved FHP. In previous studies, both CCFE and VG had an effect on the CVA $[4,8,20]$. In addition, CCFE was found to help maintain a neutral alignment of the cervical spine and significantly increase the CVA and cervical range of motion in both the sitting and standing positions in healthy subjects $[4,20]$. Additionally, CCFE increased the activation of local muscles to improve uprightness of the cervical spine and maintain position [26]. The VG might influence activity of the global and local neck muscles via movements of the eyeball $[8,25]$. Previous research indicated that patients with neck pain exhibited particular impairments that altered motor control of the deep and superficial neck muscles to support and control the cervical spine [7], and that they showed the delayed activation of both the DCFs and superficial SCM and AS and altered activation of SCM and DCFs in response to postural perturbations [22]. The CCFE-VG was designed as an exercise method for rehabilitation based on motor relearning principles. Optimization of motor relearning and synchronous co-contraction of local and global muscles are important to increasing stability [21], and coordination between the deep and superficial flexors is necessary for safe progress of exercise in patients with neck pain [4]. Moreover, CCFE-VG could be beneficial for reductions in the end-range strain and maintenance of a neutral posture of the cervical spine [22]. Additionally, mechanical stability was shown to be sustained when co-contraction between local and global muscles was performed harmoniously during functional activities [21]. Therefore, CCFE-VG may have provided stability of the cervical spine via activation of the global muscles in the neck in this study. Moreover, intervention combining VG and CCFE increased CVA relative to CCFE alone. These findings indicate that intervention using CCFE-VG could lead to greater improvements in FHP compared to CCFE alone.

The activity of the SCM and AS muscles increased significantly during CCFE-VG compared to during CCFE alone across all CCFE phases. In a previous study, CCFE improved the deep core stability in the neck by increasing the activity of DCFs in the neck [20]. Additionally, the activity of SCM during cervical rotation was dependent on the VG. That is, increased activity of the SCM was measured when the subject directed their gaze toward the direction of movement compared to when they looked away or moved the eyes with the head [23]. This finding may be explained by the reflex coordination of eye muscle activity and the neck to coordinate movement [24]. Animal studies have identified neuronal connections between eyeball and neck movement that could mediate such reflex coordination [25]. Previous human studies reported that increased neural connections (vestibule-collic reflex and vestibule-ocular reflex) could drive neck muscle activity in association with voluntary horizontal eyeball movement in the same direction $[23,24]$. In the present study, CCFE-VG led to greater activation of the SCM and AS in subjects with FHP compared to CCFE alone. In a previous investigation, CCFE was significantly associated with improvement of the ability to hold a neutral cervical posture during prolonged sitting causing neck and shoulder pain [26]. Increased activation of the SCM and AS by VG in CCFE-VG could improve stability in the cervical spine and help maintain its neutral alignment. Together, our results indicate that CCFE-VG as the motor relearning intervention could lead to greater improvement in FHP by harmonious activation of the local and global muscles in the neck compared to CCFE alone.

It should be noted that this study has several limitations. Specifically, the long-term effects of CCFE-VG could not be determined because the study was designed to investigate 
the immediate effects of CCFE-VG using a cross-sectional design. Additionally, the findings in this study cannot be generalized to other patient populations that have FHP with neck and shoulder pain. Future studies should apply CCFE-VG to subjects with FHP and pain around the neck and shoulder and examine the long-term effects of CCFE-VG. Moreover, this study could not measure the muscle activity of the deep neck flexor because it was performed by using surface EMG. Finally, although we tried to study the effects of visual gazing in CCFE, viewing angles and eyeball movements were not recorded. We assumed that subjects who participated in the study followed instructions to move the eyeball downward without flexion of the head in CCFE. Therefore, further studies should be conducted to record kinetic eyeball movement.

\section{Conclusion}

This study compared the immediate effects of CCFE and CCFE-VG on CVA and the activity of the SCM and AS muscles in subjects with FHP. CCFE-VG significantly increased CVA and the activity of the SCM and AS muscles across all CCFE phases compared to CCFE alone. The results of the present study suggest that CCFE-VG may be useful for improving FHP in subjects.

\section{References}

[1] Harman K, Hubley-Kozey CL. Butler H. Effectiveness of an exercise program to improve forward head posture in normal adults: a randomized, controlled 10-week trial. Journal of Manual \& Manipulative Therapy. 2005; 13(3):163-76.

[2] Braun BL., Amundson LR. Quantitative assessment of head and shoulder posture. Archives of Physical Medicine and Rehabilitation. 1989;70(4):322-9.

[3] Darling DW, Kraus S, Glasheen-Wray MB. Relationship of head posture and the rest position of the mandible. Journal of Prosthetic Dentistry. 1984:52(1):111-5.

[4] Jull GA, Falla D, Vicenzino B, et al. The effect of therapeutic exercise on activation of the deep cervical flexor muscles in people with chronic neck pain. Manual Therapy. 2009;14(6):696-701.

[5] Griegel-Morris P, Larson K, Mueller-Klaus K, et al. Incidence of common postural abnormalities in the cervical, shoulder, and thoracic regions and their association with pain in two age groups of healthy subjects. Physical Therapy. 1992;72(6):425-31.

[6] Darnell MW. A proposed chronology of events for forward head posture. Journal of Craniomandibular Practice. 1983;1(4):49-54.

[7] Jull GA, O'Leary SP, Falla DL. Clinical assessment of the deep cervical flexor muscles: the craniocervical flexion test. Journal of Manipulative and Physiological Therapeutics. 2008;31(7):525-33.

[8] Bexander CS, Mellor R, Hodges PW. Effect of gaze direction on neck muscle activity during cervical rotation. Experimental Brain Research. 2005;167(3):422-32.

[9] Han Y, Lennerstrand G. Eye position changes induced by neck muscle vibration in strabismic subjects. Graefe's Archive for Clinical and Experimental Ophthalmology; Heidelberg. 1999;237(1):21-8.

[10] Fernändez-De-Las-Peñas C, Pérez-De-Heredia M, MoleroSànchez A, et al. Performance of the Craniocervical Flexion Test, Forward Head Posture, and Headache Clinical Parameters in Patients With Chronic TensionType Headache: A Pilot Study. Journal of Orthopaedic \& Sports Physical Therapy. 2007;37(2):33-9.

[11] Kim KH, Kim SG, Hwangbo G. The effects of horse-riding simulator exercise and Kendall exercise on the forward head posture. Journal of Physical Therapy Science. 2015; 27(4):1125-7.

[12] Peterson DE, Blankenship KR, Robb JB, et al. Investigation of the validity and reliability of four objective techniques for measuring forward shoulder posture. 
Journal of Orthopaedic \& Sports Physical Therapy. 1997;25(1):34-42.

[13] Hallman DM, Olsson EM, Von Schéele B, et al. Effects of heart rate variability biofeedback in subjects with stress-related chronic neck pain: a pilot study. Applied Psychophysiology and Biofeedback. 2011;36(2):71-80.

[14] Yoo W, Yi C, Cho SH, et al. Effects of the height of ball-backrest on head and shoulder posture and trunk muscle activity in VDT workers. Industrial Health. 2008;46(3):289-97.

[15] Lewis JS, Wright C, Green A. Subacromial impingement syndrome: the effect of changing posture on shoulder range of movement. The Journal of Orthopaedic and Sports Physical Therapy. 2005;35(2):72-87.

[16] Criswell E. Cram's introduction to surface electromyography. Jones \& Bartlett Publishers. 2010.

[17] Kendall FP, McCreary EK, Kendall HO. Muscles, Testing and Function: Testing and Function. Lippincott Williams and Wilkins. 1983.

[18] Jull G, Trott P, Potter H, et al. A randomized controlled trial of exercise and manipulative therapy for cervicogenic headache. Spine. 2002;27(17):1835-43.

[19] Soloman SR, Sawilowsky SS. Impact of rank-based normalizing transformations on the accuracy of test scores. Journal of Modern Applied Statistical Methods. 2009;8(2):448-62.
[20] Camitsis A. The effect of craniocervical flexion exercise on cervical posture and cervical range of motion in asymptomatic participants. 2015.

[21] McGill SM, Grenier S, Kavcic N et al. Coordination of muscle activity to assure stability of the lumbar spine. Journal of Electromyography and Kinesiology. 2003; 13(4):353-9.

[22] Cheng CH, Chen TY, Kuo YW, et al. The Mechanics of Cervical Muscle Recruitment on Cervical Spine Stability -A Biomechanical in Vitro Study using Porcine Model. Journal of Mechanics. 2008;24(1):63-8.

[23] André-Deshays C, Berthoz A, Revel M. Eye-head coupling in humans. I. Simultaneous recording of isolated motor units in dorsal neck muscles and horizontal eye movements. Experimental Brain Research. 1988;69(2): 399-406.

[24] André-Deshays C, Revel M, Berthoz A. Eye-head coupling in humans. II. Phasic components. Experimental Brain Research. 1990;84(2):359-66.

[25] Berthoz A, Grantyn A. Neuronal mechanisms underlying eye-head coordination. Progress in Brain Research. 1986;64:325-43.

[26] Falla D, Jull G, Russell T, et al. Effect of Neck Exercise on Sitting Posture in Patients With Chronic Neck Pain. Physical Therapy. 2007;87(4):408-17. 